\title{
FEASIBILITY OF PHYSIOTHERAPY IN PATIENTS AFTER INTERVENTATIONAL CARDIOLOGY PROCEDURES
}

\author{
Antoaneta Dimitrova \& Zhasmina Koleva \\ National Sports Academy "Vassil Levski", Sofia, Bulgaria
}

\begin{abstract}
Introduction: The main goals of physiotherapy in elderly patients after transcatheter aortic valve implantation (TAVI) with many comorbidities are to increase the physiological response to the body's oxygen needs, to reduce the symptoms and to prevent of a secondary complications which could worsen their daily life activities.

Methodology: The purpose of the study is to evaluate the influence of the exercise therapy on a pulmonary functional status in patients three months after TAVI. 15 males and 16 females (mean age 77,6 64,3 ; NYHA classes III-IV), referred for physiotherapy before and after TAVI underwent in-hospital and after discharge four assessments to evaluate the spirometry parameters, inspiratory capacity, physical tolerance and dyspnea.

Results and discussion: The inspiratory capacity is an important indicator for respiratory dysfunction. There is an improvement of $649 \mathrm{ml}$ on the $3^{\text {rd }}$ month compared to the baseline in the mean values. All the patients had a significant improvement in respiratory function $(p<0,01)$, dyspnea $(p<0,001)$ and $6 M W T(p<0,001)$, after the three months of regular therapeutic exercise sessions. The early application of an incentive breathing device for voluntary respiratory training is applicable in patients with TAVI in clinical setting and after discharge. The improvement of respiratory function is important for the prevention of congestive pneumonia and to improve oxygen supply.

Conclusions: The physiotherapy program shows benefits in respiratory function and is suitable for the patients with TAVI.
\end{abstract}

Key words: physiotherapy, interventional cardiology

\section{INTRODUCTION}

Exercise-based cardiac rehabilitation in TAVI patients is leading to significant improvements in exercise tolerance, walking capacity, muscle strength and quality of life, and reduces frailty (Eicher, et al., 2017; Nechwatal, 2018; Russo, 2014). The patients are encouraged to participate in regular KT sessions not only in hospital but also after discharge in order to maintain and improve their functional status in daily activities. Exercise programs are pointed out as the best type of intervention to improve physical function (Scottish Intercollegiate Guidelines Network, 2016; Arantes, et al., 2009). The official position statements promote the implementation of exercise-based individualized cardiac rehabilitation after TAVI, in accordance with patient's everyday life and can be delivered as an inpatient or outpatient program (Piepoli, et al., 2016).

The purpose of the study is to evaluate the influence of the physiotherapy (PT) on a respiratory and functional status in patients three months after TAVI.

\section{METHODS}

31 patients ( 15 male, 16 female; mean age $78,4 \pm 3,1$; NYHA III-IV before TAVI) referred for PT underwent 4 assessments in-hospital and after discharge to evaluate the muscle strength and muscle endurance with the following tests - six minutes walking test (6MWT), Borg scale and spirometry. The assessments were performed before TAVI procedure, on the day of hospital discharge, on the $1^{\text {st }}$ and $3^{\text {rd }}$ month after TAVI procedure.

The 6MWT was performed in accordance with the instructions of the American Thoracic Association (ATS, 2002). The aim is to identify the changes in the physical tolerance of the patients (Butland, 1982). The Borg Scale assesses the dyspnea and fatigue during the exercising (Borg, 1982).

Exercise protocol for in-hospital physiotherapy

Exercise protocol for in-hospital PT was performed twice daily, with low intensity (40-50\% HR max), duration from $10-15 \mathrm{~min}$ to $30-45 \mathrm{~min}$, on average for 10 days. The protocol included mobilization to sitting, standing and slow walking at least six minutes daily; rhythmic exercises for the 
distal parts of the limbs, exercises for gradually strengthening the upper and the lower limbs, diaphragmatic and chest breathing, breathing with a device (Coach2 Incentive Spirometer) for inspiratory training, isometric exercises in circular mode. The feedback breathing device for inspiratory training was applied to all patients with the instruction to make 5 repetitions with 1 to $3 \mathrm{~min}$ rests between them, at least 4-5 times a day, every day of the week during hospital stay (Grigorova-Petrova, 2018).

Exercise protocol for home-based physiotherapy Home-based PT was performed twice daily, every day, for 3 months. All the patients were given a device for inspiratory training and a booklet with instructions for exercising at home with a description of all therapeutic exercises and how to increase gradually the walking distance, stairs climbing, daily activities, etc. The patients were instructed how to measure their heart rate during the exercises at home. The indications for discontinuing the exercising are arrhythmia, tachycardia, precordial pain or chest discomfort, a change in the breathing pattern with inclusion of additional respiratory muscles, severe fatigue or dyspnea, muscle weakness, patient's desire to discontinue.

\section{STATISTICAL ANALYSIS}

All statistical analyses were performed using SPSS for Windows, Version 19.0. The level of significance was set at $p$ less than 0.05 . All data subsets were assessed for the presence of a normal distribution (Shapiro-Wilk test p > 0.05) and for homogeneity with Levene's test for equality of variance before using parametric analysis. Differences between data in 6MWT, spirometric parameters and Borg Scale were assessed by repeated measures analysis of variance with post hoc multiple comparisons by t-test with Bonferroni corrections.

\section{RESULTS}

The patients were observed over the study period of 3 months (Table 2). We compared the effect of PT in patients after TAVI on walking, fatigue and respiratory function in 4 time points.

Table 1. Changes in physical activity and dyspnea (mean value and standard deviation) in the patients over the study period

\begin{tabular}{|c|c|c|c|c|c|}
\hline \multirow[b]{2}{*}{ Test } & \multicolumn{5}{|c|}{ mean values $\pm \mathrm{SD}$} \\
\hline & $\begin{array}{c}\text { before } \\
\text { TAVI }\end{array}$ & $\begin{array}{l}\text { on hospital } \\
\text { discharge }\end{array}$ & \multicolumn{2}{|c|}{$1^{\text {st }}$ month } & $3^{d}$ month \\
\hline $6 \mathrm{MWT}(\mathrm{m})$ & $102,4 \pm 73,9$ & $161,2 \pm 106,3$ & \multicolumn{2}{|l|}{$250,3 \pm 98,7$} & $295,2 \pm 73,8$ \\
\hline p-value & \multicolumn{5}{|l|}{$0,002^{\text {a }}$} \\
\hline Borg scale (points) & $6,6 \pm 2,6$ & \multirow[t]{2}{*}{$4,2 \pm 2,4$} & \multicolumn{2}{|l|}{$2,8 \pm 1,6$} & $1,9 \pm 1,4$ \\
\hline p-value & \multicolumn{4}{|l|}{$\mathbf{0 , 0 0 1}{ }^{\mathrm{a}}$} & \\
\hline FVC (1) & $1,8 \pm 0,8$ & $1,9 \pm 07$ & $2,1 \pm 0,5$ & \multicolumn{2}{|l|}{$2,2 \pm 0,6$} \\
\hline p-value & \multicolumn{5}{|l|}{$0,008^{\text {a }}$} \\
\hline $\mathrm{FEV}_{1}(1)$ & $1,4 \pm 0,5$ & $1,3 \pm 0,4$ & $1,4 \pm 0,4$ & \multicolumn{2}{|l|}{$1,5 \pm 0,4$} \\
\hline p-value & \multicolumn{5}{|l|}{$0,221^{\mathrm{a}}$} \\
\hline $\mathrm{FEV}_{1}(\%)$ & $62,1 \pm 17,6$ & $66,2 \pm 19,8$ & $81,1 \pm 10,1$ & \multicolumn{2}{|l|}{$85,9 \pm 8,1$} \\
\hline p-value & \multicolumn{5}{|l|}{$\mathbf{0 , 0 0 0}{ }^{\mathrm{a}}$} \\
\hline
\end{tabular}

TAVI-transcatheter aortic valve implantation;6MWT-6 minutes walking test; ${ }^{a}-R M$ ANOVA;SD-standard deviation; $F V C$ - forced vital capacity; $F E V_{1}$ - forced expiratory volume at one second; $F E V_{1}(\%)$ - forced expiratory volume as a percent of predicted value;

The patients had significant improvement in the physical tolerance 3 months post TAVI. The walking distance increased with $193 \mathrm{~m}$ in the mean values on the $3^{\text {rd }}$ month after TAVI (from 102. $4 \pm 73$. 9 to $295.2 \pm 73.8 \mathrm{~m}$ ). The patients had decreased the levels of dyspnoea with 4,7 points in the mean values after 3 months of KT. Their score in mean values decreased from 6,6 points (very strong dyspnea) to 1,9 points (weak dyspnea). All patients had significant improvement in physical tolerance, in FVC, in $\mathrm{FEV}_{1}$, decreased levels of dyspnea and fatigue after three months of regular PT. 


\section{DISCUSSION}

The main elements of cardiac rehabilitation include establishing an individually tailored treatment plan to help regain strength, preventing the complications after implantation, reducing the risk of future heart problems, and improving functional status and health-related quality of life. KT contributes to reduction of the physical disability these patients suffer from (Cribier, et al., 2002; Fauchere, et al., 2014; Lawrie, 2012).

The $6 \mathrm{MWT}$ has been shown to be responsive to clinically important changes in symptoms and health status in patients with chronic pulmonary, neurological or cardiac diseases (Gremeaux, et al., 2011; Grigorova-Petrova, 2017; Lyubenova, 2015; Puhan, et al., 2011; Redelmeier, et al., 1997; Shoemaker, et al., 2012; Wise, et al., 2005). Although $6 \mathrm{MWT}$ has been used for years as an estimate of cardiopulmonary reserve, walking speed is a summary indicator of overall physical vitality because walking integrates the circulatory, respiratory, nervous, and musculoskeletal systems (Dimitrova, et al., 2017; Ross, et al., 2010; Studenski, et al., 2011). Most of the conducted studies compare the effect of $\mathrm{KT}$ on the functional status in patients after TAVI versus after surgical aortic valve replacement (Russo, et al., 2014; Yue, et al., 2015; Fauchère, et al., 2012; Zanettini, et al., 2014; Green, et al., 2013). In the research of Fauchère, et al., (2012) the TAVI patients were older and with a higher morbidity rate than the patients which underwent a surgical aortic valve replacement. The authors concluded that the patients that underwent TAVI benefit in the same way after a rehabilitation program according $6 \mathrm{MWT}$ and Functional Independence Measure scores. A recent study of 484 patients with severe symptomatic aortic stenosis who underwent TAVI, evaluated the association between physical performance as estimated by the 6MWT and long-term prognosis after TAVI. The authors reported that the patients who were unable to walk experienced a higher rate of death after TAVI compared with the "fast walkers" whose distance was above the median. Another conclusion was that the patients who were unable to walk and those that were slow walkers at the beginning experienced an improvement in functional status after TAVI, whereas the fast walkers did not improve but experienced a modest decrease in six minutes walking distance instead. The 6MWT was lower than predicted for healthy older adults, both at baseline and during follow-up due to the severe aortic stenosis, advanced age, comorbidities and lower activities of everyday living (Green, et al., 2013). Casanova, et al., (2011) concluded that the $10 \%$ of 70 - to 80 -year-old healthy males and females walked above $400 \mathrm{~m}$ in six minutes. Enright, et al., (2003) derived a formula for predicting $6 \mathrm{MWT}$ among healthy older adults. The formula includes gender, age, height and weight. The predicted normal lower limit for women and men should be approximately 230 and $250 \mathrm{~m}$. In a study of patients with chronic heart failure, changes in $6 \mathrm{MWT}$ of 25 to $50 \mathrm{~m}$ were associated with clinically meaningful changes in health status (O'Keeffe, et al., 1998). A large controlled trial with 2,054 subjects investigated the outcomes of exercise training (mean age 59 years, 36\% with NYHA class III or IV heart failure symptoms). The mean value of the $6 \mathrm{MWT}$ was $372 \mathrm{~m}$ and $75 \%$ of patients had distances of more than $300 \mathrm{~m}$ (Forman, et al., 2012). Gotzmann, et al., (2010) performed 6MWT on 44 participants at baseline and 1 month after TAVI and found that median 6MWT improved after TAVI. In two studies, Bagur, et al., (2011) performed 6MWT at baseline and 6 months after TAVI and demonstrated that $25 \%$ of subjects did not improve their walking distance at follow-up compared with baseline. The author reported that $30 \%$ of patients did not demonstrate functional improvement 6 months after TAVI assessed by Duke Activity Status Index questionnaire. Mok, et al., (2013) demonstrated an association between baseline 6MWT and all-cause mortality among 212 TAVI patients who were able to perform the test at a single center (mean 6MWT $182 \mathrm{~m}$ ). The high mortality rates after TAVI among patients that participated in the PARTNER trial ( $43.3 \%$ at 2 years) have motivated clinicians to attempt to identify patients that would benefit from TAVI in regards to their functional status and quality of life (Makkar, et al., 2012). Green, et al., (2013) suggest that even in patients that cannot walk before the intervention, it a TAVI procedure is worth performing. Those that survive after TAVI had a great improvement in walking distance. A decrease in walking distance after TAVI had been seen among the fastest walkers at baseline.

Inspiratory training involves the training of the specific muscles including diaphragma and intercostal muscles. Feedback inspiratory training devices are used for improving pulmonary function and providing feedback about respiratory training to the patients with different diseases (Dimitrova, 
et al., 2017; Grigorova-Petrova et al., 2014). The early application of an incentive breathing device for voluntary respiratory training is applicable in patients with TAVI in clinical setting and after discharge. The improvement of respiratory function is important for prevention of congestive pneumonia and to improve oxygen supply.

\section{CONCLUSION}

Physiotherapy after TAVI improves the physical tolerance and respiratory function of the elderly patients. The levels of dyspnea and fatigue decreased after 3 months of PT.

\section{REFERENCES}

Arantes, P., Alencar, M., Dias, R. et al. (2009). Physical therapy treatment on frailty syndrome: systematic review. Rev Bras Fisioter., Vol 13 No.5, pp. 365-375

ATS, American Thoracic Society Committee on Proficiency Standards for Clinical Pulmonary Function Laboratories. (2002). ATS Statement: guidelines for the 6-minute walk test. Am J Respir Crit Care Med., No.166, pp. 111-117.

Bagur, R., Rodes-Cabau, J., Dumont, E., et al. (2011). Exercise capacity in patients with severe symptomatic aortic stenosis before and six months after transcatheter aortic valve implantation. Am J Cardiol., No.108, pp. 258-264.

Bagur, R., Rodes-Cabau, J., Dumont, E. et al. (2011). Performance-based functional assessment of patients undergoing transcatheter aortic valve implantation. $A m$ Heart J., No.161, pp. 726-734.

Borg, G. (1982). Psychophysical bases of perceived exertion. Med Sci Sports Exerc., No.14, pp. 377-381.

Butland, R., Pang, J., Gross, E., et al. (1982). Two-, sixand 12 minute walking tests in respiratory disease. $B M J$, No. 284, pp. 1607-1608.

Casanova, C., Celli, B., Barria, P. et al. (2011). The 6-min walk distance in healthy subjects: reference standards from seven countries. Euro Resp J., No.37, pp.150-156.

Cribier, A., Eltchaninoff, H., Bash, A. et al. (2002). Percutaneous transcatheter implantation of an aortic valve prosthesis for calcific aortic stenosis. Circulation, No.106, pp. 3006-8

Dimitrova, A., Izov, N., Maznev, I., Vasileva, D., Nikolova, M. (2017). Physiotherapy in patients with chronic obstructive pulmonary disease. Open Access Macedonian Journal of Medical Sciences, Vol 5 No. 6, pp.720-723

Eichler, S., Salzwedel, A., Reibis, R., et al. (2017). Multicomponent cardiac rehabilitation in patients after tran- scatheter aortic valve implantation: Predictors of functional and psychocognitive recovery. Eur J Prev Cardiol., No. 24, pp. 257-264

Enright, P., McBurnie, M., Bittner, V. et al. (2003). The 6-min walk test: a quick measure of functional status in elderly adults. Chest, No.123, pp.387-398.

Fauchere, I., Weber, D., Maier, W. et al. (2014). Rehabilitation after TAVI compared to surgical aortic valve replacement. International Journal of Cardiology, Vol 173 No.3, pp.564-566

Forman, D., Fleg, J., Kitzman, D. et al. (2012). 6-min walk test provides prognostic utility comparable to cardiopulmonary exercise testing in ambulatory outpatients with systolic heart failure. J Am Coll Cardiol., No.60, pp.26532661

Gotzmann, M., Hehen, T., Germing, A. et al. (2010). Short-term effects of transcatheter aortic valve implantation on neurohormonal activation, quality of life and 6-minute walk test in severe and symptomatic aortic stenosis. Heart, No.96, pp.1102-1106.

Green, P., Cohen, D., Genereux, P., et al. (2013). Relation between six-minute walk test performance and outcomes after transcatheter aortic valve implantation (from the PARTNER trial), Am J Cardiol, Vol. 112, pp. 700-706

Gremeaux, V., Troisgros, O., Benaim, S. et al. (2011). Determining the minimal clinically important difference for the six-minute walk test and the 200-meter fast-walk test during cardiac rehabilitation program in coronary artery disease patients after acute coronary syndrome. Arch Phys Med Rehab., No. 92, pp.611-619.

Grigorova-Petrova K, Lubenova D, Dimitrova A, Baldaranov D, Lozeva J. (2014). Feasibility of early physical therapy program in-hospital patients with acute ischemic stroke, Maced J Med Sci., Vol 2 No. 3, pp. 452-455

Grigorova-Petrova, K. (2017). Izsledvane i otsenka na motornoto vŭzstanovyavane pri patsienti sŭs sledinsultni sǔstoyaniya. Sport i nauka, No.6, pp.54-59

Grigorova-Petrova, K. (2018). Vliyanie na inspiratornata trenirovka vŭrkhu podvizhnostta na diafragmata pri patsienti sŭs sledinsultni sŭstoyaniya. Sport $i$ nauka, No.6, pp. 95-103

Lawrie, G. (2012). Role of Transcatheter Aortic Valve Implantation (TAVI) Versus Conventional Aortic Valve Replacement in the Treatment of Aortic Valve Disease. Methodist Debakey Cardiovasc J., Vol 8 No.2, pp. 4-8 Lyubenova, D. (2015). Vliyanie na kineziterapiyata pri bolni s mozŭchen insult. Sport i nauka, No.5, pp.104117.

Makkar, R., Fontana, G., Jilaihawi, H. et al. (2012). Transcatheter aortic-valve replacement for inoperable severe 
aortic stenosis. N Engl J of Med., No.366, pp.1696-1704

Mok, M., Nombela-Franco, L., Urena, M. et al. (2011). Pre-procedural six-minute walk test as a predictor of mortality in patients undergoing transcatheter aortic valve implantation. J Am Coll Cardiol., No.61, pp. $897-$ 898.

O’Keeffe, S., Lye, M., Donnellan, C., Carmichael, D. (1998). Reproducibility and responsiveness of quality of life assessment and six minute walk test in elderly heart failure patients. Heart, No.80, pp. 377-382.

Nechwatal, R. (2018). Cardiac rehabilitation after surgical and transcatheter valve replacement and repair. Dtsch Z Sportmed. No. 69, pp. 285-292.

Piepoli, M., Hoes, A., Agewall, S. (2016). European Guidelines on cardiovascular disease prevention in clinical practice: the Sixth Joint Task Force of the European Society of Cardiology and Other Societies on Cardiovascular Disease Prevention in Clinical Practice (constituted by representatives of 10 societies and by invited experts): developed with the special contribution of the European Association for Cardiovascular Prevention and Rehabilitation (EACPR). Eur J Prev Cardiol., 2016; No.23, pp. NP1-NP96.

Puhan, M., Chandra, D., Mosenifar, Z., Ries, A. et al. (2011). National Emphysema Treatment Trial Research Group. The minimal important difference of exercise tests in severe COPD. Eur Respir J., No.37, pp.784-790.

Redelmeier, D., Bayoumi, A., Goldstein, R., Guyatt, G. (1997). Interpreting small differences in functional sta- tus: the six minute walk test in chronic lung disease patients. Am J Respir Crit Care Med., No.155, pp.1278-1282

Ross, R., Murthy, J., Wollak, I., Jackson, A. (2010). The six minute walk test accurately estimates mean peak oxygen uptake. BMC Pulm Med., No.10, pp. 31-40.

Russo, N., Compostella, L., Tarantini, G., et al. (2014). Cardiac rehabilitation after transcatheter versus surgical prosthetic valve implantation for aortic stenosis in the elderly. European Journal of Preventive Cardiology, Vol 21 No.11, pp. 1324-1331

Scottish Intercollegiate Guidelines Network (SIGN). Cardiac rehabilitation. Edinburgh: SIGN; 2016. (SIGN publication no. 150). [July 2017]. http://www.sign.ac.uk Studenski, S., Perera, S., Patel, K. et al. (2011). Gait speed and survival in older adults. JAMA, No.305, pp. 50-58.

Yue, Li. (2015). Improvement in Quality of Life in Old People with Aortic Stenosis after Transcatheter Aortic Valve Implantation, Current Signal Transduction Therapy,Vol.9 No.3, pp.164-171

Zanettini, R., Gatto, G., Mori, I. et al. (2014). Cardiac rehabilitation and mid-term follow-up after transcatheter aortic valve implantation. Journal of Geriatric Cardiolo$g y$, No.11, pp. 279-285

Corresponding author: Antoaneta Dimitrova, M.D., Ph.D.

Department of Physiotherapy and Rehabilitation National Sports Academy "Vassil Levski”, Sofia, Bulgaria Email: tonialllex@yahoo.com 